\title{
La cirugía bariátrica modifica la diversidad bacteriana y los metabolitos de la microbiota intestinal mejorando el perfil metabólico del huésped y contribuyendo a la reducción del peso.
}

\section{Bariatric surgery modifies the bacterial diversity and metabolites of the gut microbiota by improving the metabolic profile of the host and contributing to weight reduction.}

\author{
Roberto E.Vigil-Guerrero', Manuel Gasco², \\ Gustavo F. Gonzales ${ }^{2}$
}

\author{
Vigil-Guerrero RE, Gasco M, Gonzales GF. La cirugía bariátrica modifica \\ la diversidad bacteriana y los metabolitos de la microbiota intestinal \\ mejorando el perfil metabólico del huésped y contribuyendo a la \\ reducción del peso. Rev Soc Peru Med Interna. 2021;34(4):162-172. \\ DOI: https://doi.org/10.36393/spmi.v34i4.632
}

RESUMEN

La obesidad es una enfermedad crónica multifactorial de características inflamatorias que afecta a ambos sexos y a todas las edades a nivel mundial, aumentando la morbilidad y mortalidad por diversas enfermedades. La obesidad se asocia a disbiosis de la microbiota intestinal, alterando con ello la absorción de nutrientes y el metabolismo energético. La cirugía bariátrica ha demostrado ser el mejor tratamiento para la obesidad mórbida y las enfermedades asociadas al sobrepeso. Los estudios de los efectos de la cirugía bariátrica sobre la microbiota intestinal, realizados en los últimos cinco años, se caracterizaron por tener poca cantidad de sujetos en las muestras, con seguimientos entre seis meses y un año, teniendo resultados heterogéneos. En general, la cirugía bariátrica produce cambios importantes en la microbiota intestinal, con aumento de los filos Proteobacteria, Fusobacteria y Verrucomicrobia; y disminución del filo Firmicutes. Akkermansia muciniphila puede ser una bacteria-clave asociada a los beneficios obtenidos por la cirugía. La diversidad bacteriana aumenta a partir de los seis meses de la cirugía, y la conformación final de la microbiota, luego de un periodo de adaptación, está asociado a un perfil metabólico bacteriano detox-redox con poca liberación de energía. No se ha demostrado ninguna relación de causalidad entre los cambios de la microbiota intestinal producidos por la cirugía y los efectos beneficiosos de la misma, aunque los estudios de trasplante de material fecal sugieren una verdadera transferencia fenotípica asociada al peso y al perfil metabólico. Conocer los mecanismos de esta relación microbiota-hospedero ayudaría a encontrar intervenciones terapéuticas con los mismos resultados que se obtienen con la cirugía. En conclusión, la cirugía bariátrica induce cambios importantes en la microbiota intestinal, donde los metabolitos bacterianos interactúan con el huésped mejorando el perfil metabólico y contribuyendo a la pérdida del peso.

Palabras claves: cirugía bariátrica, microbiota intestinal, obesidad. 


\begin{abstract}
Obesity is a chronic multifactorial disease with inflammatory characteristics that affects both sexes and all ages worldwide, increasing morbidity and mortality from various diseases. Obesity is associated with dysbiosis of the gut microbiota, thereby altering nutrient absorption and energy metabolism. Bariatric surgery has proven to be the best treatment for morbid obesity and diseases associated with overweight. Studies of the effects of bariatric surgery on the gut microbiota conducted in the last five years were characterized by a small number of subjects in the samples and a follow-up between 6 months and I year, with heterogeneous results. In general, bariatric surgery produces important changes in the intestinal microbiota, with an increase in Proteobacteria, Fusobacteria and Verrucomicrobia phyla; and decrease of the phylum Firmicutes. Akkermansia muciniphila it can be a key bacteria associated with the benefits obtained by surgery. The bacterial diversity increases from 6 months after surgery, and the final composition of the microbiota, after a period of adaptation, is associated with little energy release and detox-redox profile in the bacterial metabolism. No proven causal relationship between changes in the microbiota due to surgery and the beneficial effects in the host, although fecal material transplantation studies suggest a true phenotypic transfer associated with weight and metabolic profile. Knowing the mechanisms of this microbiota-host relationship would help to find therapeutic interventions with the same results that are obtained with surgery. In conclusion, bariatric surgery induces important changes in the gut microbiota, where bacterial metabolites interact with the host by improving metabolic profile and contributing to weight loss.
\end{abstract}

Keywords: bariatric surgery, gut microbiome, obesity.

\section{INTRODUCCIÓN}

La obesidad es una enfermedad crónica, multifactorial, de características inflamatorias, que aumenta la morbilidad y mortalidad cuando se asocia a otras enfermedades, tales como diabetes, hipertensión, esteatosis hepática, dislipidemia, síndrome metabólico. Se presenta en cualquier lugar del mundo, y en todas las edades, con una prevalencia ligeramente más alta en las mujeres que en los hombres. ${ }^{1}$ La ingesta de comidas ricas en carbohidratos y grasas, asociado a poca actividad física, generan un desbalance entre el aporte y el gasto calórico, que producen un aumento en el peso corporal por acumulación de tejido graso, afectando el metabolismo de lípidos y carbohidratos. ${ }^{2}$ El contenido y la distribución de la grasa corporal total son rasgos sexualmente dimórficos. Las mujeres tienen un mayor contenido de grasa corporal que los hombres, especialmente en la región glúteo-femoral (obesidad ginecoide). Los hombres presentan mayor masa muscular y la acumulación de grasa es más abdominal y visceral (obesidad androide), que a su vez son los más nocivos para la salud. Con la menopausia las mujeres comienzan a acumular grasa en el compartimiento visceral, con lo que aumenta su riesgo de morbilidad y mortalidad a enfermedades crónicas no transmisibles. ${ }^{3}$

Si bien una de las estrategias que se ha desarrollado en las últimas décadas para reducción de peso es la cirugía bariátrica, los hombres representan una minoría entre los pacientes que se someten a este tipo de cirugía. Generalmente tienen un índice de masa corporal (IMC) más alto y con mayores comorbilidades que las mujeres. El beneficio del procedimiento es igual en ambos sexos, aunque la satisfacción es mayor en los hombres, necesitándose más estudios sobre los aspectos específicos del sexo en el resultado de la cirugía bariátrica, especialmente en lo que respecta a los aspectos metabólicos. ${ }^{4}$

La presente es una revisión narrativa donde se intenta analizar cómo es que la cirugía bariátrica produce reducción de peso afectando el metabolismo de los lípidos y carbohidratos. Para ello se revisó la literatura de los últimos cinco años donde se aborda la asociación de la cirugía bariátrica con la microbiota intestinal, y si tiene un rol importante en el metabolismo calórico-proteico del hospedero.

\section{MICROBIOTA INTESTINAL}

La microbiota intestinal es la población microbiana que vive en el intestino de manera comensal y mutualística con el hospedero. Está compuesto principalmente por bacterias, además de arqueas, hongos y virus. ${ }^{5}$ El término microbioma se refiere al número total de microorganismos y su material genético, referido no solo a la cantidad y composición, sino también a los metabolitos que producen y a las funciones que ejercen en el medio intestinal. ${ }^{6}$

El microbioma humano ha ido evolucionando con los seres y a lo largo del tiempo, desarrollándose comunidades microbianas específicas en lugares anatómicos específicos en el cuerpo. ${ }^{7}$ La colonización con organismos comensales comienza en el feto por una transmisión vertical de la madre demostrado por la presencia de microorganismos en la placenta, en el líquido amniótico, en la sangre del cordón umbilical y en el meconio; después, con el nacimiento, de acuerdo al tipo de parto, por la exposición a la microbiota vaginal. ${ }^{8}$ A partir de ese momento, factores del medio ambiente, como la dieta y la interrelación con otros seres humanos, van definiendo el perfil de la microbiota, alcanzando su máxima diversidad en la adolescencia, manteniéndose estable hasta las últimas etapas de la vida. Varios aspectos de la vida, como el uso de antibióticos, las infecciones y la estructura de la dieta producen cambios profundos y duraderos sobre el microbioma humano. ${ }^{9-11}$ La microbiota intestinal de cada ser humano es única y es considerada como una "huella digital".

Se calcula que la cantidad de bacterias en el intestino es, como mínimo, igual a la cantidad de células humanas en el cuerpo (proporción 1:1), con una presencia genómica 
Tabla I. Metabolitos producidos por la microbiota intestinal.

\begin{tabular}{|c|c|c|c|}
\hline Metabolito & Sustrato & Receptores & Acción \\
\hline \multirow{2}{*}{$\begin{array}{l}\text { AGCC (butirato, propionato, } \\
\text { acetato) }\end{array}$} & \multirow{2}{*}{ Fibras en la dieta } & \multirow{2}{*}{ GPR-4I/GPR-43 células L } & $\begin{array}{l}\text { Estimula la producción de GLP-I, } \\
\text { PYY, Pro-glucagón. }\end{array}$ \\
\hline & & & Estimula la proliferación de células $L$. \\
\hline \multicolumn{4}{|l|}{ Aminoácidos: } \\
\hline - BCCA y $\operatorname{ImP}$ & Histidina & & Aumenta la resistencia a la insulina. \\
\hline \multirow{3}{*}{ - indol y derivados } & \multirow{3}{*}{ Triptofano } & \multirow{3}{*}{$\begin{array}{l}\text { Expresión de los receptores } \\
\text { de hidrocarburos de arilo }\end{array}$} & Aumentan GLP-I \\
\hline & & & $\begin{array}{l}\text { Mejoran la función de la barrera } \\
\text { intestinal }\end{array}$ \\
\hline & & & Disminuye la inflamación \\
\hline \multirow{2}{*}{-TMA } & \multirow{2}{*}{ Colina y L carnitina } & \multirow{2}{*}{ TMAO hepático } & $\begin{array}{l}\text { Riesgo cardiovascular } \\
\text { (arterioesclerosis) }\end{array}$ \\
\hline & & & $\begin{array}{l}\text { Hiperrespuesta plaquetaria y } \\
\text { trombosis }\end{array}$ \\
\hline \multicolumn{4}{|l|}{ Neurotransmisores: } \\
\hline \multirow{2}{*}{$\begin{array}{l}\text { - clásicos: histamina, serotonina, } \\
\text { GABA, catecolaminas }\end{array}$} & \multirow{2}{*}{ Histidina, L-glutamato, cisteina. } & \multirow{2}{*}{ Células L } & Regulación de la inmunidad intestinal. \\
\hline & & & Cognición- Conducta. \\
\hline - gaseosos: $\mathrm{NO}, \mathrm{H} 2 \mathrm{~S}$ & $\begin{array}{l}\text { Nitrógeno, oxígeno, hidrógeno, } \\
\text { metano, monóxido de carbono. }\end{array}$ & Células L & Cambios en la motilidad intestinal \\
\hline $\mathrm{N}$-acyl-amidas & Lípidos & GPR-I I 9 células L & Mejoran la tolerancia a la glucosa \\
\hline \multicolumn{4}{|l|}{ Componentes Bacterianos: } \\
\hline - ClpB & proteína bacteriana & $\begin{array}{l}\text { Similar a hormona } \\
\text { estimulante de melanocitos. }\end{array}$ & $\begin{array}{l}\text { Modulación de los patrones de } \\
\text { alimentación. }\end{array}$ \\
\hline \multirow{2}{*}{-Amuc_II00 } & \multirow{2}{*}{ proteína bacteriana } & \multirow{2}{*}{ TLR-2 } & Mejora la barrera intestinal. \\
\hline & & & Reduce peso y masa grasa. \\
\hline \multicolumn{4}{|l|}{ Ácidos Biliares: } \\
\hline Primarios & & FXR nuclear & Aumenta la reisistencia a la insulina. \\
\hline $\begin{array}{l}\text { Secundarios (ácido deoxicólico, } \\
\text { ácido litocólico) }\end{array}$ & Ácidos biliares primarios & TGR5 (GPCR) & $\begin{array}{l}\text { Mejora la sensibilidad a la insulina. } \\
\text { En el tejido graso marrón incrementa } \\
\text { el gasto de energía y protege contra } \\
\text { la obesidad. }\end{array}$ \\
\hline
\end{tabular}

AGCC: ácido graso cadena corta. BCCA: aminoácido esencial ramificado. ImP: propionato de Imidazol.TMA: trimetilamina. TMAO: óxido de trimetilamina. GABA: ácido gamaaminobutírico. NO: óxido nítrico. H2S: ácido sulfíhidrico. Receptores: GPR, TGR, AhR, TLR, FXR.Adaptado de Rastelli et al 2019.

100 veces mayor. ${ }^{12}$ La mayor proporción son bacterias que se encuentran en el colon, distribuidas en los filos Firmicutes y Bacteroidetes, que son la mayoría, además de los filos Proteobacteria, Actinobacteria, Fusobacteria y Verrucomicrobia. ${ }^{13,14}$ Las funciones centrales de la microbiota intestinal saludable incluyen la biodegradación de polisacáridos, la producción de ácidos grasos de cadena corta, el enriquecimiento de lipopolisacáridos específicos y la producción de vitaminas y aminoácidos esenciales. ${ }^{15}$ (Tabla 1).

\section{DISBIOSIS}

La variación interindividual hace difícil definir una única microbiota saludable. ${ }^{6}$ El retorno a una conformación microbiana previa a la enfermedad podría estar relacionado a la recuperación de la salud (resiliencia bacteriana). ${ }^{16}$ Cuando se altera la microbiota intestinal ocurre una disbiosis. La disbiosis es la incapacidad de retornar a un estado de microbiota saludable y está asociada a la disminución de la diversidad bacteriana, inflamación y aumento de la permeabilidad intestinal, lo que a su vez impacta negativamente sobre la microbiota, alterando la absorción de nutrientes y el metabolismo energético. ${ }^{17}$

Los sujetos obesos tienen una microbiota intestinal disbiótica. ${ }^{18}$ La obesidad materna durante el embarazo es acompañada por disbiosis intestinal que se transmite en forma vertical al feto, induciéndole disbiosis intestinal y desórdenes metabólicos. ${ }^{19}$ Estos cambios tienen relación con el proceso inflamatorio de bajo grado producido por el aumento del peso, en especial del compartimiento graso 
(lipotoxicidad), y se asocia a resistencia a la insulina y, en casos graves, a síndrome metabólico y esteatosis hepática no alcohólica. ${ }^{20}$

En general, la disbiosis asociada a la obesidad se caracteriza, a nivel de filo, por un aumento de Firmicutes y una disminución de Bacteroidetes, aunque en la actualidad se reconoce que el reacomodo de las comunidades bacterianas para producir el mismo efecto metabólico varía de individuo en individuo. ${ }^{21}$ Asimismo, en los sujetos obesos, la abundancia relativa de la familia Christensenellaceae y los géneros Methanobacteriales, Lactobacillus, Bifidobacteria y Akkermansia, todos ellos usados como probióticos, se asocian inversamente con obesidad. ${ }^{17} \mathrm{La}$ microbiota intestinal modula la obesidad por la regulación de la absorción de energía de los alimentos, del apetito central, de los depósitos grasos, de la inflamación crónica y del ritmo circadiano. ${ }^{17}$ (Tabla 2).

\section{MICROBIOTA INTESTINAL Y TRATAMIENTO ANTI- OBESIDAD}

La modificación en la microbiota intestinal que producen los tratamientos de la obesidad, tanto el tratamiento clínico como el quirúrgico, señalizan vías metabólicas beneficiosas en el control del peso y las enfermedades asociadas. ${ }^{22} \mathrm{El}$ tratamiento clínico de la obesidad consiste en cambios de estilo de vida (dietas y actividad física) y/o uso de fármacos que han conseguido buenos resultados a corto plazo. ${ }^{23}$

\section{Cirugía bariátrica}

El tratamiento quirúrgico, conocido como cirugía bariátrica, se ha convertido en una opción terapéutica con buenos resultados a largo plazo, tanto en el control del peso como de las enfermedades asociadas, disminuyendo la morbimortalidad y mejorando la calidad de vida. ${ }^{24} \mathrm{La}$ cirugía bariátrica consiste en la reducción del estómago, asociado o no, a una alteración de la anatomía del intestino proximal denominado bypass. La técnica patrón de oro es el bypass gástrico en $\mathrm{Y}$ de Roux (BG), aunque hoy en día la técnica que más se hace en el mundo es la manga gástrica o gastrectomía tipo Sleeve (MG). ${ }^{25}$ (Tabla 3).

La cirugía bariátrica produce un re-arreglo de la anatomía gastrointestinal que provoca la disminución de la ingesta de alimentos y altera la producción de entero hormonas. ${ }^{26}$ Estos efectos son mediados por la microbiota intestinal ${ }^{27} \mathrm{y}$ las sales biliares. ${ }^{28}$ Las modificaciones de cada uno de estos factores provoca, a su vez, cambios metabólicos que van a afectar al huésped. ${ }^{29}$

Cirugía barátrica y cambios en la microbiota intestinal Los trabajos de los últimos cinco años sobre el tema, se caracterizaron por tener muestras pequeñas, la mayoría no aleatorias, y con un tiempo de seguimiento no mayor a un año. ${ }^{30-54}$ En los resultados se aprecia que después de la cirugía bariátrica hubo cambios importantes de la microbiota intestinal, la mayoría similares en ambas técnicas (BG y MG).

A nivel de filo aumentaron Fusobacteria, Proteobacteria y Verrucomicrobiota; y disminuyó Firmicutes. A nivel de Clase aumentaron Bacilli y Gammaproteobacteria. A nivel de Orden aumentó Lactobacillales. A nivel de Familia aumentaron Enterobacteriaceae, Rikenelliaceae y Streptococcaceae, y disminuyeron Bacteroidaceae y Bifidobacteriaceae. A nivel de Género aumentaron Actinomyces, Aggregatibacter, Akkermansia, Alistipis, Anaerotroncus, Clostridium, Granulicatella, Haemophylus, Klebsiella, Lachnospiaceae sp., Odoribacter, Prevotella, Rothia, Ruminococcus, Streptococcus y Veillonella; y disminuyeron Bifidobacterium y Coprococcus. A nivel de

TABLA 2. Mecanismos de obesidad inducidos por la microbiota intestinal

\begin{tabular}{|c|c|c|}
\hline \multicolumn{2}{|l|}{ EFECTO } & \multirow{2}{*}{$\begin{array}{l}\text { MICROBIOTA } \\
\begin{array}{l}\text { Expresión de genes (CD36) que controlan la absorción } \\
\text { de lípidos aumentada }\end{array}\end{array}$} \\
\hline $\begin{array}{l}\text { MAYOR ABSORCIÓN DE } \\
\text { ENERGÍA }\end{array}$ & Aumenta Desulfovibrio y disminuye Clostridia & \\
\hline $\begin{array}{l}\text { ENERGÍA EXTRA PARA EL } \\
\text { HUÉSPED }\end{array}$ & $\begin{array}{l}\text { Asociación inversa entre AGCC fecal y diversidad } \\
\text { microbiana intestinal.Aumento de Faecalibacterium } \\
\text { prausnitzii, Roseburia faecis, y otros Clostridiales. } \\
\text { Disminución de Akkermansia muciniphila, Alistipes } \\
\text { finegoldi, Bacteroides, Christensenellaceae, Methano- } \\
\text { brevibacter, y Oscillospira. }\end{array}$ & Exceso de AGCC \\
\hline AUMENTO DEL APETITO & Comunidad disminuida de Clostridial XIVa y VI & Disminución de PYY y GLPI \\
\hline $\begin{array}{l}\text { AUMENTO DEL DEPÓSITO } \\
\text { GRASO }\end{array}$ & Disminución de Lactobacillus paracasei & $\begin{array}{l}\text { Aumento de la expresión de ChREBP y SREBPI, Fiaf es } \\
\text { inhibido, LPL activado, triglicéridos hepáticos entran a la } \\
\text { circulación. Disminución de la expresión de ANGPTL4 } \\
\text { (inhibidos de lipoproteinalipasa. }\end{array}$ \\
\hline INFLAMACIÓN CRÓNICA & Disminucion de Ruminococcaceae y Lachnospiraceae. & $\begin{array}{l}\text { Disminución de butirato que es un metabolito anti-in- } \\
\text { flamatorio. Butirato estimula adipoliolisis, fosforilacion } \\
\text { oxidativa mitocondrial, y reduce lipopolisacáridos. }\end{array}$ \\
\hline $\begin{array}{l}\text { INTERRUPCIÓN DEL CICLO } \\
\text { CIRCADIANO }\end{array}$ & $\begin{array}{l}\text { Alteración de Lachnospiraceae, Clostridiaceae, } \\
\text { Ruminococcaceae, Lactobacillus, Bacteroides y Bifido- } \\
\text { bacterium. }\end{array}$ & $\begin{array}{l}\text { Participan en la biotransformación de ácidos biliares. } \\
\text { Regulan la transcripción de genes envueltos en el ciclo } \\
\text { circadiano (Dbp, Per I/2) y el metabolismo de lípidos } \\
\text { (Ppargamma,Angptl4) }\end{array}$ \\
\hline
\end{tabular}

Adaptado de Liu BN et al 2021. 
Tabla 3. Características por tipo de cirugía bariátrica.

\begin{tabular}{|c|c|c|}
\hline & MANGA GÁSTRICA (sleeve) (MG) & BYPASS GÁSTRICO (BG) \\
\hline \multirow{2}{*}{ MECANISMO } & \multirow{2}{*}{ Restrictivo } & Restrictivo \\
\hline & & Malabsorcion \\
\hline \multirow{3}{*}{ RESULTADOS } & Reduce la cantidad de comida ingerida & Reduce la cantidad de comida ingerida \\
\hline & Efecto anorexigénico & Disminuye la absorción proximal de alimentos \\
\hline & Efecto metabólico sobre la glucosa y lípidos & $\begin{array}{l}\text { Efecto metabólico incretínico independiente de la } \\
\text { pérdida del peso }\end{array}$ \\
\hline \multirow{4}{*}{ VENTAJAS } & Técnica simple con tiempo corto de cirugía & Resultados se mantienen en el tiempo \\
\hline & Indicada en pacientes de alto riesgo clínico para la cirugía de bypass & Remisión de enfermedades asociadas a la obesidad \\
\hline & Primer paso de cirugía en superobesos & \multirow{2}{*}{ Patrón oro de la cirugía bariátrica/metabólica } \\
\hline & Efectiva para controlar peso y enfermedades asociadas & \\
\hline \multirow{5}{*}{ DESVENTAJAS } & Procedimiento no reversible & Técnica más compleja que la manga o la banda gástrica \\
\hline & Aumenta el reflujo gastroesofágico & Deficiencias de vitaminas y minerales \\
\hline & \multirow{3}{*}{ Efecto metabólico menor a las técnicas de bypass } & Riesgo de complicaciones a nivel intestinal \\
\hline & & Riesgo de úlcera gastrojeyeyunal \\
\hline & & Síndrome de dumping \\
\hline
\end{tabular}

Adaptado de American Society for Metabolic and Bariatric Surgery (ASMBS)

Tabla 4. Cambios de la microbiota intestinal después de la cirugía (BG y MG).

\begin{tabular}{|c|c|c|c|c|c|c|}
\hline & PHYLUM & CLASE & ORDEN & FAMILIA & GENERO & ESPECIES \\
\hline \multirow{19}{*}{ AUMENTA } & Fusobacteria & & & & & \\
\hline & \multirow{4}{*}{ Proteobacteria* } & \multirow{4}{*}{ Gammaproteobacteria* } & & \multirow{4}{*}{ Enterobacteriaceae* } & Aggregatibacter & \\
\hline & & & & & Haemophilus* & \\
\hline & & & & & Klebsiella* & \\
\hline & & & & & Haemophilus & Haemophilus parainfluenzae \\
\hline & Verrucomicrobia* & & & & Akkermansia* & Akkermansia muciniphila* \\
\hline & & \multirow{3}{*}{ Bacilli $(F)^{*}$} & \multirow{3}{*}{ Lactobacillales* } & \multirow{3}{*}{ Streptococcaceae* } & Granulicatella* & \\
\hline & & & & & \multirow{2}{*}{ Streptococcus* } & Streptococcus parasanguinis* \\
\hline & & & & & & Streptococcus salivarius* \\
\hline & & & & Rikenellaceae (B) & Alistipes* & Alistipes finegoldii \\
\hline & & & & & Actinomyces $(A)$ & \\
\hline & & & & & Rothia* (A) & \\
\hline & & & & & Odoribacter (B) & \\
\hline & & & & & Prevotella (B) & \\
\hline & & & & & Anaerotruncus (F) & \\
\hline & & & & & Clostridium (F) & \\
\hline & & & & & $\begin{array}{l}\text { Lachnospiraceae } \\
\text { sp (F) }\end{array}$ & \\
\hline & & & & & Ruminococcus* $(F)$ & \\
\hline & & & & & Veilloneia* $(F)$ & \\
\hline \multirow{3}{*}{ DISMINUYE } & Firmicutes* & & & & Coprococcus & \\
\hline & & & & $\begin{array}{l}\text { Bifidobcteriaceae } \\
(A)^{*}\end{array}$ & Bifidobacterium* & \\
\hline & & & & Bacteroidaceae (B) & & Bacteroides stercoris \\
\hline
\end{tabular}

(*) Bacterias más mencionadas en los estudios. F: firmicutes, B: bacteroidetes, A: actinobacteria, P: proteobacteria, F: fusobacteria,V: verrucomicrobia. 
Tabla 5. Cambios adicionales de la microbiota intestinal por tipo de cirugía.



Especies aumentaron Akkermansia muciniphila, Alistipes finegoldi, Haemophilus parainfluenzae, Streptococcus parasanguinis y Streptococcus salivarius; y disminuyó Bacteroides stercoris (Tabla 4).

Hay alteraciones específicas de acuerdo al tipo de cirugía. En el BG aumentaron los géneros Enterococcus y Escherichia, y disminuyeron los géneros Blautia, Faecalibacterium y Roseburia, y la especie Lachnospiraceae spp. En la MG aumentó la especie Bacteroides uniformis, y disminuyeron las especies Bacteroides fragilis, Dorea longicatena $y$ Ruminococcus gnavus. (Tabla 5)

La diversidad microbiana disminuye después de la cirugía bariátrica y comienza a aumentar a partir del tercer mes post quirúrgico, manteniéndose estable en el tiempo de estudio de la mayoría de los trabajos. ${ }^{30-35,37,40-42,44,46,49-51,53,54}$ En los primeros meses después de la cirugía, quizás por tratarse de un periodo de adaptación a los cambios anatómicos y fisiológicos, hay menos cantidad de nutrientes y con diferente composición (menos carbohidratos y grasas), lo que hace que prevalezcan microbiotas más preparadas para extraer energía de alimentos no digeridos en momentos de gran restricción calórica, como las Enterobacteriaceae (Proteobacteria)..$^{33,34,40}$

Stefura y col. encontraron que los sujetos que consiguieron perder $50 \%$ o más del exceso de peso a los seis meses de la cirugía, tuvieron en la microbiota basal preoperatoria abundancia de Epsilonproteobacteria (Proteobacteria), y los que no lo consiguieron tuvieron abundancia de Rikenellaceae (Bacteroidetes) y Lachnospiraceae (Firmicutes). ${ }^{55}$ En un trabajo posterior ${ }^{56}$, al igual que Ilhan y col. ${ }^{57}$, encontraron que el perfil de la microbiota intestinal seis meses después de la cirugía, era distinto en los sujetos que consiguieron perder peso comparado con los que no lo consiguieron, pero con presencia de géneros y especies diferentes. Pajecki y col. encontraron que el paciente con la peor pérdida del exceso de peso $(50.79 \%)$ a los seis meses de la cirugía, tuvo disminución de Bacteroidetes y aumento de Firmicutes, perfil parecido al de la obesidad. ${ }^{36}$

Estos resultados pueden servir de guía para la selección de pacientes que van a ser sometidos a cirugía bariátrica con la finalidad de mejorar la tasa de éxito; sin embargo, queda aún por dilucidar qué se debe hacer en los casos que el perfil de la microbiota no sea adecuado para una cirugía bariátrica efectiva.

\section{Efectos metabólicos de los cambios de la microbiota intestinal sobre el huésped}

Las alteraciones del $\mathrm{pH}$ intraluminal, por la modificación del estómago y el uso de inhibidores de bomba de protones, y la cantidad de oxígeno que llega al intestino terminal, son más pronunciados en el BG que en la MG. Este medio puede favorecer la presencia de bacterias orales anaerobias facultativas, ácidos sensibles, bilio-resistentes, como Fusobacteria, Granulicatella, Odoribacter, Streptococcus y Veillonella. Por otro lado, puede inhibir la presencia de bacterias anaerobias obligatorias como Blautia, Roseburia, Faecalibacterium y Bifidobacterium..$^{30,34,38,43}$ Blautia está asociada a individuos con diabetes mellitus. ${ }^{33} \mathrm{~F}$. prausnitzii produce metabolitos que bloquean la secreción de mediadores inflamatorios, y también inducen la secreción de GLP1 mejorando la sensibilidad a la insulina, y Roseburia, que aumenta tardíamente después de la cirugía, algunos estudios la relacionan a la remisión de la diabetes mellitus. $^{37}$

Bacterias del orden Clostridiales, y Prevotella (Bacteroidetes), todas productoras de butirato, comienzan a aumentar después de la cirugía. ${ }^{31,32,49}$ Streptococcus y Lactococcus, productores de lactato, han sido usados como probióticos para mejorar la integridad intestinal, desde que el lactato es crucial para los productores de butirato, y por consiguiente de la salud colónica. ${ }^{35,53}$

Hungatella hathewayi (orden Clostridiales) se asocia a la disminución de la masa grasa del tronco y la hemoglobina glicosilada. ${ }^{34}$ Alistipes shahii (familia Rikenellaceae), se asocia con mejora metabólica. La disminución de los niveles de bacterias de la familia Rikenellaceae está asociada a enfermedad hepática no alcohólica (NAFL). ${ }^{50}$ Bacteroides uniformis, formador de biofilm, aumenta en 
MG y disminuye en BG. B. uniformis está asociado a la reestructuración de la colonización normal del intestino y reduce la producción de citoquinas inflamatorias. ${ }^{33,44}$ Por el contrario, Bacteroides que liberan sulfatos durante la degradación de la mucina, y especies de la familia Dethosulfovibrionacea, como Bilophila sp, que reducen el sulfato produciendo sulfuro de hidrógeno con efectos inflamatorios, al igual que Acidaminococcus y Lachnospira, se correlacionan negativamente con la pérdida de peso, y están asociados a complicaciones de la obesidad, como síndrome metabólico y diabetes. ${ }^{45}$ Ruminococcus gnavus y $R$. torques, que disminuyen después de la cirugía, producen transcialidasa para degradar mucina y están asociados a inflamación y desórdenes metabólicos. ${ }^{34}$

Después de la cirugía se han podido observar dos bacterias potencialmente dañinas, relacionadas a enfermedad de colon: Yokenella regensburgei (Proteobacteria) y Fusobacterium varium. La primera aparece a los tres meses y se mantiene hasta los seis meses post cirugía, y está asociada a inflamación. La segunda se asocia a cáncer de colon. ${ }^{39}$

Otras bacterias reaccionan de manera diferente de acuerdo a si se encuentran en un medio disbiótico o normal. $B$. Thetaiotaomicron se asocia a la presencia de $B$. uniformis para producir un efecto sinergístico en la disminución de peso, en la medida que el primero aumenta el contenido graso en roedores libre de gérmenes. ${ }^{46}$ Anaerostipes hadrus, productora de butirato, exacerba la colitis inducida en roedores, pero que en animales sanos se relaciona a resultados favorables. ${ }^{42}$ Akkermancia muciniphila (Verrucromicrobia) aumenta con ambas cirugías. Bacteria capaz de degradar mucina, mejorar la barrera intestinal, formar bio-films y contribuir al metabolismo de lactato. Se relaciona a la pérdida de peso, disminución de la adiposidad, mejor perfil metabólico con disminución de la resistencia a la insulina, y efectos antiinflamatorios. Se asocia a remisión de diabetes mellitus. Puede aumentar con metformina y puede mediar esta acción..$^{34,35,37,38,43,48,53}$ Mabey y col. describieron altas tasas de A. municiphila en un paciente con remisión de la diabetes mellitus después del tratamiento clínico (sin cirugía). ${ }^{33}$

Paganelli y col. al comparar las dos cirugías, sin diferencias en la composición basal de la microbiota intestinal y con resultados clínicos similares, sugieren que la cirugía bariátrica, por sí misma, induce una alteración de la composición de la microbiota a largo plazo, independiente de las modificaciones de la dieta en el post operatorio. ${ }^{40}$ Shen y col., al comparar dos grupos de obesos mórbidos de regiones geográficas diferentes (New York y Barcelona), no encontraron diferencias postoperatorias en los resultados clínicos ni en la composición de la microbiota intestinal, por lo que sugieren que la cirugía, en estas condiciones específicas de obesidad severa, podría contrarrestar las influencias genéticas, ambientales o dietéticas sobre el microbioma. ${ }^{41}$

La cirugía bariátrica produce cambios en el metabolismo

\section{microbiano intestinal}

En muchos estudios la alteración en las vías metabólicas y funciones del microbioma han sido configurados usando KEGG (Kyoto Encyclopedia of Gens and Genomas). ${ }^{58}$ KEGG usa una base de datos que asigna significados funcionales a genes y genomas usando ortólogos para predecir cambios funcionales de alto nivel en la forma de mapas de vías metabólicas.

Los microbios pueden actuar sinérgicamente en la producción y degradación de metabolitos, reflejando interacciones entre especies que son fisiológicamente importantes, efecto que aparece muy temprano después de la cirugía41. Un factor muy importante es la interrelación de la microbiota intestinal con los ácidos biliares. Las bacterias lo usan como sustrato de biotransformación en el colon, produciéndose una verdadera comunicación entre ambos, con efectos beneficiosos en el metabolismo del huésped a través de los receptores Farnesoide X y TGR5. ${ }^{35,38}$

En una revisión encontraron que la microbiota intestinal post operatoria de sujetos con obesidad mórbida fue similar a la de sujetos con sobrepeso o con obesidad leve, con aumento de GLP1, GLP2, PYY y TMAO y disminución de BCCA (aminoácidos ramificados esenciales), que en su conjunto dieron como resultado la disminución de glucosa, insulina, triglicéridos, colesterol total, LDL, HOMA IR, disminución del apetito, y remisión de diabetes mellitus. ${ }^{59}$ Samszuck y col. encontraron, a los 6 meses de la cirugía, cambios metabólicos similares en ambas técnicas (BG y $\mathrm{MG}$ ), como el metabolismo de aminoácidos y fosfolípidos, $\mathrm{y}$ otros que eran diferentes, como el metabolismo de sustratos que contienen sulfuro en el BG y el metabolismo de $\mathrm{p}$-cresol y carbohidratos en la $\mathrm{MG}$, en pacientes con los mismos resultados clínicos post operatorios. ${ }^{60}$

LI y col. concluyeron que el BG induce un conjunto de efectos que incluyen el metabolismo de aminoácidos (tirosina, fenialalanina, triptofano y metionina) y ácidos grasos mitocondriales, lo que podría contribuir no solo a la pérdida de peso y al efecto antidiabético, sino también a procesos metabólicos externos al sistema digestivo. ${ }^{51}$ Ellos sugieren que los cambios en la microbiota intestinal contribuyen al co-metabolismo bacteria-huésped probablemente dirigidos por las comunidades bacterianas favorecidas por las condiciones de la cirugía. Sánchez y col. concluyeron que la capacidad de las bacterias colónicas para extraer energía del azúcar es más baja después de la cirugía como consecuencia de la reducción del número de rutas por las cuales la microbiota puede metabolizar azúcar. ${ }^{47}$ Este sistema detox-redox post cirugía, de menor generación de energía, podría ser la base para la pérdida de peso y el control metabólico de la diabetes.

\section{Trasplante de material fecal (TMF)}

El trasplante de material fecal (TMF) consiste en la administración de una solución de material fecal debidamente procesada, procedente de un donante sano, en el tracto gastrointestinal de otro individuo, preferencialmente por 
sonda nasogástrica/nasoyeyunal o por colonoscopía, con el fin de manipular las características de la microbiota del receptor. ${ }^{61}$ También puede ser administrado con cápsulas vía oral. ${ }^{62} \mathrm{El}$ TMF tiene buenos resultados comprobados en el tratamiento de la diarrea refractaria por Clostridium difficile. ${ }^{63}$ Varios estudios están evaluando el papel del TMF en diferentes enfermedades, en especial enfermedad inflamatoria intestinal y síndrome metabólico. ${ }^{64-66}$

No se ha podido determinar una relación de causalidad entre los beneficios de la cirugía bariátrica y las alteraciones que produce en la microbiota intestinal. ${ }^{67} \mathrm{El}$ TMF sugiere un posible efecto causal de la alteración de la microbiota intestinal sobre el peso y el metabolismo del receptor, mucho más claro en estudios experimentales en roedores que en estudios clínicos en humanos.

Los trabajos de Liou y col. y Tremaroli y col. sugieren una transferencia, vía microbiota, de los efectos beneficiosos sobre el peso y la adiposidad, de los donadores a los receptores. ${ }^{68,69}$ Tremaroli trasplantó material fecal de sujetos obesos post operados de cirugía bariátrica a roedores libres de gérmenes obteniendo menor adiposidad y peso en los receptores; y, Liou trasplantó material fecal de roedores obesos a roedores libres de gérmenes obteniendo el efecto contrario. La transferencia en bloque de la microbiota intestinal humana contenida en el material fecal de un donador magro saludable a un receptor obeso transfiere temporalmente el fenotipo beneficioso del primero al segundo, mejorando la adiposidad y el peso. El TMF de donadores magros mejora la sensibilidad de la insulina en receptores obesos con síndrome metabólico, y las mejores respuestas se encuentran cuando la microbiota basal del receptor presenta riqueza genética microbiana alta o cuando el donador está metabólicamente saludable. ${ }^{70}$

\section{CONCLUSIONES}

Desde que se describió que las mejoras metabólicas después de la cirugía bariátrica eran independientes de la pérdida de peso, se han descrito diferentes mecanismos de acción de los diferentes factores que participan en este proceso. Uno de los factores más estudiados en estos últimos años es la microbiota intestinal.

La cirugía bariátrica modifica de manera importante la microbiota intestinal: aumenta la diversidad y altera la abundancia relativa de algunas especies y grupos bacterianos, conformando una comunidad cuyos metabolitos interactúan con el huésped para mejorar el perfil metabólico, especialmente de los carbohidratos y los lípidos, contribuyendo al control de la glicemia y del peso. Los cambios en la microbiota intestinal podrían deberse a un conjunto de factores asociados a los cambios anatómicos de la cirugía bariátrica. La restricción de la dieta, el aumento del tránsito intestinal, los cambios intraluminales del $\mathrm{pH}$ y de los niveles de oxígeno, la alteración de los ácidos biliares, la disminución del área de absorción de nutrientes y la digestión retrasada al yeyuno distal-íleon proximal, inducen cambios en la conformación y metabolismo bacterianos que, a través de un complejo sistema de interacción, modula el metabolismo del huésped.

La cirugía bariátrica induce un aumento de los filos Fusobacteria, Proteobacteria y Verrucomicrobia; y una disminución del filo Firmicutes. Akkermansia muciniphila podría ser una de las bacteria-clave presentes en la microbiota intestinal post cirugía bariátrica que se asocia con la disminución del peso y el control glicémico del huésped.

El metabolismo bacteriano probablemente en los primeros tres meses sea de adaptación, luego del cual consigue una estabilidad en el tiempo, caracterizándose por un sistema detox-redox con menor generación de energía, lo que podría explicar los resultados metabólicos postoperatorios. El trasplante de material fecal sugiere una relación de causaefecto entre las comunidades microbianas trasplantadas y los efectos metabólicos y/o terapéuticos que producen en el receptor, pudiendo ser una verdadera transferencia de fenotipo. Este trasplante de heces transfiere la microbiota completa, es decir, no solo las bacterias, sino también virus, hongos y arqueas, por lo que sería interesante conocer el rol que juegan cada uno de estos grupos microbianos y su relación con el hospedero. Por otro lado, parece que la conformación de una comunidad bacteriana (diversidad y abundancias relativas) es más importante que la participación individual de las mismas, en la medida que bacterias con efectos nocivos en una determinada situación tienen efectos beneficiosos en otra situación, como, por ejemplo, el medio intraluminal colónico después de la cirugía bariátrica.

Los resultados heterogéneos de los estudios clínicos sobre microbiota intestinal pueden deberse a variaciones entre los sujetos, como etnia, sexo, región geográfica; a variaciones en el proceso de toma de muestra y conservación de las mismas; a variaciones en los métodos utilizados para el estudio taxonómico y metabólico de las bacterias; a variaciones en las asociaciones de los metabolitos bacterianos con los metabolitos del huésped provenientes del suero, los tejidos o las heces; y, a variaciones de los métodos estadísticos utilizados.

Dilucidar los mecanismos de acción puede tener muchas ventajas en el campo terapéutico de la obesidad y las enfermedades asociadas al sobrepeso, como mejorar el tratamiento clínico farmacológico y no farmacológico, determinar en el preoperatorio los posibles resultados del tratamiento quirúrgico, y mantener los resultados de la cirugía evitando el aumento del peso y el retorno de las enfermedades asociadas.

De la presente revisión narrativa se concluye que la cirugía bariátrica induce cambios importantes en la microbiota intestinal, donde los metabolitos bacterianos interactúan con el huésped mejorando el perfil metabólico y contribuyendo a la pérdida del peso.

\section{REFERENCIAS BIBLIOGRÁFICAS}

I. Jaacks LM,Vandevijvere S, Pan A, McGowan CJ,Wallace C, Imamura F, Mozaffarian D, Swinburn B, Ezzati M.The obesity transition: stages of the global epidemic. Lancet Diabetes Endocrinol. 2019 Mar;7(3):231240. doi: 10.1016/S2213-8587(19)30026-9. 
2. Apovian CM. Obesity: definition, comorbidities, causes, and burden. Am J Manag Care. 2016 Jun;22(7 Suppl):s I76-I85.

3. Le Magueresse-Battistoni B.Adipose tissue and endocrine-disrupting chemicals: does sex matter? Int J Environ Res Public Health. 2020 Dec I5; I 7(24):9403. doi: 10.3390/ijerph I 7249403.

4. Jäger P,Wolicki A, Spohnholz J, Senkal M. Review: Sex-specific aspects in the bariatric treatment of severely obese women. Int J Environ Res Public Health. 2020 Apr 15;17(8):2734. doi: 10.3390/ ijerph I7082734. PMID: 3232659l.

5. Riccio P, Rossano R. The human gut microbiota is neither an organ nor a commensal. FEBS Lett. 2020 Oct;594(20):3262-327I. doi:I0.1002/I873-3468.13946

6. Shanahan F, Ghosh TS, O'Toole PW. The healthy microbiome-what is the definition of a healthy gut microbiome? Gastroenterology. 202 I Jan; I60(2):483-494. doi:10.1053/j.gastro.2020.09.057

7. Nodari R, Drancourt M, Barbieri R. Paleomicrobiology of the human digestive tract:A review. Microb Pathog. 202 I Aug; I 57: 104972. doi:I0.1016/j.micpath.202I.104972

8. Tochitani S.Vertical transmission of gut microbiota: Points of action of environmental factors influencing brain development. Neurosci Res. 202I Jul;168:83-94. doi:I0.1016/j.neures.2020.II.006

9. Schwartz DJ, Langdon AE, Dantas G. Understanding the impact of antibiotic perturbation on the human microbiome. Genome Med. 2020 Sep 28; I 2(1):82. doi: I0.I |86/s | 3073-020-00782-x

10. Tan GSE, Tay HL, Tan SH, Lee TH, Ng TM, Lye DC. Gut microbiota modulation: implications for infection control and antimicrobial stewardship. Adv Ther. 2020 Oct;37(I0):4054-4067. doi:I0.1007/ s|2325-020-0|458-z

II. Alemao CA, Budden KF, Gomez HM, Rehman SF, Marshall JE, Shukla SD, et al. Impact of diet and the bacterial microbiome on the mucous barrier and immune disorders. Allergy. 2021 Mar;76(3):7| 4-734. doi: I0.I I I I/all. I4548

12. Sebastián Domingo JJ, Sánchez C. From the intestinal flora to the microbiome. Rev Espanola Enfermedades Dig Organo Of Soc Espanola Patol Dig. 20 I8 Jan; I I O(I):5I doi: I0.17235 / reed.20I7.4947/20 I7

I3. Eckburg PB, Bik EM, Bernstein CN, Purdom E, Dethlefsen L, Sargent $M$, et al. Diversity of the human intestinal microbial flora. Science. 2005 Jun I0;308(5728):I635-8. doi: I0. II26/science. I I 1059I

14. Kijng CH, Desai H, Sylvetsky AC, LoTempio J, Ayanyan S, Carrie J, et al. Baseline human gut microbiota profile in healthy people and standard reporting template. PLoS One. 2019 Sep I I; |4(9):e0206484. doi: I0.137|/journal.pone.0206484

15. Rastelli M, Cani PD, Knauf C. The gut microbiome influences host endocrine functions. Endocr Rev. 2019 Oct I;40(5):|27|-84. soi: $10.1210 /$ er.2018-00280

16. Dogra SK, Doré J, Damak S. Gut microbiota resilience: Definition, link to health and strategies for intervention. Front Microbiol. 2020 Sep 15; I I:57292 I. doi: I0.3389/fmicb.2020.57292 I

17. Liu BN, Liu XT, Liang ZH,Wang JH. Gut microbiota in obesity. World J Gastroenterol. 2021 Jul 7;27(25):3837-3850. doi:I0.3748/wjg.v27. i25.3837

18. Gasmi A, Mujawdiya PK, Pivina L, Do-a A, Semenova Y, Benahmed AG, et al. Relationship between gut microbiota, gut hyperpermeability and obesity. Curr Med Chem. 202I;28(4):827-839. doi: I0.21 74/09298 6732766620072 II603I3

19. Zhou L, Xiao X.The role of gut microbiota in the effects of maternal obesity during pregnancy on offspring metabolism. Biosci Rep. 2018 Apr 13;38(2):BSR2017|234. doi:I0.I042/BSR2017/234

20. Li R, Li Y, Li C, Zheng D, Chen P. Gut microbiota and endocrine disorder. Adv Exp Med Biol. 2020; |238:|43-64. doi: I0. I007/978-98 I I5-2385-4_9

21. 2020-03-02-Conferencia-D.-Santiago-Vega Microbiota-intestinal. pdf [Internet]. [cited 202I Jun 26]. Available from: http://racve.es/ files/2020/03/2020-03-02-Conferencia-D.-Santiago-Vega_ Microbiota-intestinal.pdf

22. Muscogiuri G, Cantone E, Cassarano S, Tuccinardi D, Barrea L, Savastano S, et al. Gut microbiota: a new path to treat obesity. Int J Obes Suppl. 2019 Apr;9(I): I0-9. doi:10.1038 / s41367-019-00 I I-7

23. Pontiroli AE, Ceriani V, Tagliabue E. Compared with Controls, Bariatric Surgery Prevents Long-Term Mortality in Persons with
Obesity Only Above Median Age of Cohorts: a Systematic Review and Meta-Analysis. Obes Surg. 2020 Jul;30(7):2487-96. doi:I0.1007/ sI I695-020-04530-3

24. Kolotkin RL,Andersen JR.A systematic review of reviews: exploring the relationship between obesity, weight loss and health-related quality of life. Clin Obes. 2017 Oct;7(5):273-89. doi:10.1III/ cob. 12203

25. Bariatric Surgery Procedures |ASMBS [Internet]. American Society for Metabolic and Bariatric Surgery. [cited 202I Jun 26]. Available from: https://asmbs.org/patients/bariatric-surgery-procedures

26. Moffett RC, Docherty NG, le Roux CW.The altered enteroendocrine reportoire following roux-en-Y-gastric bypass as an effector of weight loss and improved glycaemic control. Appetite. 2021 Jan I; I 56: I04807. doi: I 0. I 0 I6/j.appet.2020. I04807

27. Lupien-Meilleur J, Andrich DE, Quinn S, Micaelli-Baret C, St-Amand $\mathrm{R}$, Roy $\mathrm{D}$, et al. Interplay between gut microbiota and gastrointestinal peptides: potential outcomes on the regulation of glucose control. Can J Diabetes. 2020 Jun;44(4):359-67. doi:10.1016/j.jcjd.2019.10.006

28. Mazidi M, de Caravatto PPP, Speakman JR, Cohen RV. Mechanisms of action of surgical interventions on weight-related diseases: the potential role of bile acids. Obes Surg. 2017 Mar;27(3):826-36. doi: 10.1007/s I I695-017-2549-I

29. Jain AK, le Roux CW, Puri P, Tavakkoli A, Gletsu-Miller N, Laferrère $B$, et al. Proceedings of the 2017 ASPEN Research Workshop-Gastric Bypass: Role of the Gut. JPEN J Parenter Enteral Nutr. 2018 Feb;42(2):279-95. doi: 10.1002 / jpen.II2I

30. Steinert RE, Rehman A, Souto Lima EJ, Agamennone V, Schuren FHJ Gero D, et al. Roux-en-Y gastric bypass surgery changes fungal and bacterial microbiota in morbidly obese patients-A pilot study. PloS One. 2020; I 5(7):e0236936. doi: I 0. I37I/journal.pone.0236936

3I. Yu D, Shu X-O, Howard EF, Long J, English WJ, Flynn CR. Fecal metagenomics and metabolomics reveal gut microbial changes after bariatric surgery. Surg Obes Relat Dis Off J Am Soc Bariatr Surg. 2020 Nov; I6(I I): I 772-82. doi: I0.1016/j.soard.2020.06.032

32. Chen G, Zhuang J, Cui Q, Jiang S, Tao W, Chen W, et al. Two bariatric surgical procedures differentially alter the intestinal microbiota in obesity patients. Obes Surg. 2020 Jun;30(6):2345-6I. doi: 10.1007 /s I I695-020-04494-4

33. Mabey JG, Chaston JM, Castro DG, Adams TD, Hunt SC, Davidson LE. Gut microbiota differs a decade after bariatric surgery relative to a nonsurgical comparison group. Surg Obes Relat Dis Off J Am Soc Bariatr Surg. 2020 Sep;I6(9):I304-II. doi:I0.1016/j. soard.2020.04.006

34. Farin W, Oñate FP, Plassais J, Bonny C, Beglinger C, Woelnerhanssen $B$, et al. Impact of laparoscopic Roux-en-Y gastric bypass and sleeve gastrectomy on gut microbiota: a metagenomic comparative analysis. Surg Obes Relat Dis Off J Am Soc Bariatr Surg. 2020 Jul; I6(7):852-62. doi:10.1016/j.soard.2020.03.014

35. Ilhan ZE, DiBaise JK, Dautel SE, Isern NG, Kim Y-M, Hoyt DW, et al Temporospatial shifts in the human gut microbiome and metabolome after gastric bypass surgery. NPJ Biofilms Microbiomes. 2020 Mar 13;6(I): I2. doi: 10.1038 / s4 I522-020-0122-5

36. Pajecki D, de Oliveira LC, Sabino EC, de Souza-Basqueira M, Dantas $A C B$, Nunes GC, et al. Changes in the intestinal microbiota of superobese patients after bariatric surgery. Clin Sao Paulo Braz. 2019;74:e I 98. doi: I0.606 I / clinics / 2019 / el I 98

37. Lee CJ, Florea L, Sears CL, Maruthur N, Potter J], Schweitzer M, et al. Changes in gut microbiome after bariatric surgery versus medical weight loss in a pilot randomized trial. Obes Surg. 2019 Oct;29(I0):3239-45. doi: 10.1007 / s I |695-019-03976-4

38. Sánchez-Alcoholado L, Gutiérrez-Repiso C, Gómez-Pérez AM, García-Fuentes E, Tinahones FJ, Moreno-Indias I. Gut microbiota adaptation after weight loss by Roux-en-Y gastric bypass or sleeve gastrectomy bariatric surgeries. Surg Obes Relat Dis Off J Am Soc Bariatr Surg. 2019 Nov;15(II):1888-95. doi: 10.1016/ j.soard. 2019.08.55।

39. Palmisano S, Campisciano G, Silvestri M, Guerra M, Giuricin M, Casagranda B, et al. Changes in Gut Microbiota Composition after Bariatric Surgery: a New Balance to Decode. J Gastrointest Surg Off J Soc Surg Aliment Tract. 2020 Aug;24(8):1736-46. doi: 10.1007 / 
sl|605-0|9-0432|-x

40. Paganelli FL, Luyer M, Hazelbag CM, Uh H-W, Rogers MRC,Adriaans D, et al. Roux-Y Gastric Bypass and Sleeve Gastrectomy directly change gut microbiota composition independent of surgery type. Sci Rep. 2019 Jul 29;9(I):I0979. doi: I0.1038 / s4I598-019-47332-z

4I. Shen N, Caixàs A,Ahlers M, Patel K, Gao Z, Dutia R, et al. Longitudinal changes of microbiome composition and microbial metabolomics after surgical weight loss in individuals with obesity. Surg Obes Relat Dis Off J Am Soc Bariatr Surg. 2019 Aug; I 5(8): |367-73. doi: I0.1016 / j.soard.2019.05.038

42. Aron-Wisnewsky J, Prifti E, Belda E, Ichou F, Kayser BD, Dao MC, et al. Major microbiota dysbiosis in severe obesity: fate after bariatric surgery. Gut. 2019 Jan;68(I):70-82. doi: I0.1 I36 / gutjnl-2018-3 I6I03

43. Kikuchi R, Irie J, Yamada-Goto N, Kikkawa E, Seki Y, Kasama K, et al. The impact of laparoscopic sleeve gastrectomy with duodenojejunal bypass on intestinal microbiota differs from that of laparoscopic sleeve gastrectomy in Japanese patients with obesity. Clin Drug Investig. 2018 Jun;38(6):545-52. doi: I0. 1007 / s4026 I-0 I80638-0

44. Campisciano G, Palmisano S, Cason C, Giuricin M, Silvestri M, Guerra M, et al. Gut microbiota characterisation in obese patients before and after bariatric surgery. Benef Microbes. $2018 \mathrm{Apr}$ 25;9(3):367-73. doi: I0.3920 / BM20I7.0I52

45. Sanmiguel CP, Jacobs J, Gupta A, Ju T, Stains J, Coveleskie K, et al. Surgically induced changes in gut microbiome and hedonic eating as related to weight loss: preliminary findings in obese women undergoing bariatric surgery. Psychosom Med. 2017 Oct;79(8):880-7. doi: I0.1097 / PSY.0000000000000494

46. Liu R, Hong J, Xu X, Feng Q, Zhang D, Gu Y, et al. Gut microbiome and serum metabolome alterations in obesity and after weight-loss intervention. Nat Med. 2017 Jul;23(7):859-68. doi: I0.1038 / nm.4358

47. Sanchez-Carrillo S, Ciordia S, Rojo D, Zubeldia-Varela E, MéndezGarcía C, Martínez-Martínez M, et al. A body weight loss- and health-promoting gut microbiota is established after bariatric surgery in individuals with severe obesity.J Pharm Biomed Anal. 202I Jan 30; I 93: I I3747. doi: I0.1016 / j.jpba.2020.1 I3747

48. Medina DA, Pedreros JP, Turiel D, Quezada N, Pimentel F, Escalona $A$, et al. Distinct patterns in the gut microbiota after surgical or medical therapy in obese patients. PeerJ. 2017;5:e3443. doi: 10.7717 /peerj.3443

49. Lin BY, Lin W-D, Huang C-K, Hsin M-C, Lin W-Y, Pryor AD. Changes of gut microbiota between different weight reduction programs. Surg Obes Relat Dis Off J Am Soc Bariatr Surg. 2019 May; I 5(5):749-58. doi: $10.1016 /$ j.soard.2019.01.026

50. Wang F-G, Bai R-X,Yan W-M,Yan M, Dong L-Y, Song M-M. Differential composition of gut microbiota among healthy volunteers, morbidly obese patients and post-bariatric surgery patients. Exp Ther Med. 2019 Mar; I7(3):2268-78. doi: I0.3892 / etm.2019.7200

5I. Li JV, Ashrafian H, Sarafian M, Homola D, Rushton L, Barker G, et al. Roux-en-Y gastric bypass-induced bacterial perturbation contributes to altered host-bacterial co-metabolic phenotype. Microbiome. 202 I Jun I4;9(I): I 39. doi: I0. I I86 / s40 I68-02 I01086-x

52. Tabasi M,Eybpoosh S, Siadat SD, Elyasinia F, Soroush A, Bouzari S. Modulation of the gut microbiota and serum biomarkers after laparoscopic sleeve gastrectomy: a I-year follow-up study. Obes Surg. 202I May;3I(5):1949-56. doi: 10.1007 / sl |695-020-05 |39-2

53. Fouladi F, Carroll IM, Sharpton TJ, Bulik-Sullivan E, Heinberg L, Steffen KJ, et al. A microbial signature following bariatric surgery is robustly consistent across multiple cohorts. Gut Microbes. 2021 Dec;13(I):1930872. doi: 10.1080 / I9490976.2021.1930872

54. Ben Izhak M, Eshel A, Cohen R, Madar-Shapiro L, Meiri H, Wachtel $\mathrm{C}$, et al. Projection of gut microbiome pre- and post-bariatric surgery to predict surgery outcome. mSystems. 202 I Jun 29;6(3):e0 I 36720. doi: I0.I I 28 / mSystems.0 I 367-2

55. Stefura T, Zapała B, Gosiewski T, Krzysztofik M, Skomarovska
O, Major P. Relationship between bariatric surgery outcomes and the preoperative gastrointestinal microbiota: a cohort study. Surg Obes Relat Dis Off J Am Soc Bariatr Surg. 2021 May; I7(5):889-99. doi: 10.1016 / j.soard.2021.01.01 I

56. Stefura T, Zapała B, Stój A, Gosiewski T, Skomarovska O, Krzysztofik $M$, et al. Does postoperative oral and intestinal microbiota correlate with the weight-loss following bariatric surgery?-A cohort study.J Clin Med. 2020 Nov 27;9( I 2):E3863. doi: $10.3390 /$ jcm91 23863

57. Ilhan ZE, DiBaise JK, Isern NG, Hoyt DW, Marcus AK, Kang D-W, et al. Distinctive microbiomes and metabolites linked with weight loss after gastric bypass, but not gastric banding. ISME J. 2017 Sep; I I (9):2047-58. doi: I0. 1038 / ismej.20 I 7.7I

58. Ogata H, Goto S, Sato K, Fujibuchi W, Bono H, Kanehisa M. KEGG: Kyoto Encyclopedia of Genes and Genomes. Nucleic Acids Res. 1999 Jan I;27(I):29-34. doi: 10.1093 / nar / 27.1.29

59. Magouliotis DE, Tasiopoulou VS, Sioka E, Chatedaki C, Zacharoulis D. Impact of Bariatric surgery on metabolic and gut microbiota profile: A systematic review and metaanalysis. Obes Surg. 2017 May;27(5): I345- I357. doi: I0. 1007 / sl 1695-0|7-2595-8

60. Samczuk P, Luba M, Godzien J, Mastrangelo A, Hady HR, Dadan J, et al. "Gear mechanism" of bariatric interventions revealed by untargeted metabolomics. J Pharm Biomed Anal. 2018 Mar 20;151:219-226. doi: 10.1016 / j.jpba.2018.01.016

61. Vindigni SM, Surawicz CM. Fecal Microbiota Transplantation. Gastroenterol Clin North Am. 2017 Mar;46(I):I7I-I85. doi: 10.1016/j.gtc.2016.09.012

62. Gulati M, Singh SK, Corrie L, Kaur IP, Chandwani L. Delivery routes for faecal microbiota transplants:Available, anticipated and aspired. Pharmacol Res. 2020 Sep;159:104954. doi: 10.1016 / j.phrs.2020.104954

63. Tixier EN, Verheyen E, Ungaro RC, Grinspan AM. Faecal microbiota transplant decreases mortality in severe and fulminant Clostridioides difficile infection in critically ill patients. Aliment Pharmacol Ther. 2019 Nov;50(10):10941099. doi: I0. I I I I / apt. I5526

64. Green JE, Davis JA, Berk M, Hair C, Loughman A, Castle D, et al. Efficacy and safety of fecal microbiota transplantation for the treatment of diseases other than Clostridium difficile infection: a systematic review and meta-analysis. Gut Microbes. 2020Nov9; I 2(I): I-25.doi: I0.1080/ 19490976.2020.1854640

65. Colman RJ, Rubin DT. Fecal microbiota transplantation as therapy for inflammatory bowel disease: a systematic review and meta-analysis. J Crohns Colitis. 2014 Dec;8(I2):I569-8I. doi: 10.1016 / j.crohns.2014.08.006

66. de Groot P, Scheithauer T, Bakker GJ, Prodan A, Levin E, Khan MT, et al. Donor metabolic characteristics drive effects of faecal microbiota transplantation on recipient insulin sensitivity, energy expenditure and intestinal transit time. Gut. 2020 Mar;69(3):502-5I2. doi: I0.II36 / gutjnl2019-318320

67. Walter J, Armet AM, Finlay BB, Shanahan F. Establishing or exaggerating causality for the gut microbiome: Lessons from human microbiota-associated rodents. Cell. 2020 Jan 23; I80(2):22 I-232. doi: 10.1016 / j.cell.2019.12.025

68. Liou AP, Paziuk M, Luevano JM Jr, Machineni S, Turnbaugh PJ, Kaplan LM. Conserved shifts in the gut microbiota due to gastric bypass reduce host weight and adiposity. Sci Transl Med. 20I3 Mar 27;5(I78):I78ra4I. doi: I0.II26 / scitranslmed.3005687

69. Tremaroli V, Karlsson F, Werling M, Ståhlman M, Kovatcheva- 
Datchary P, Olbers T, et al. Roux-en-Y gastric bypass and vertical banded gastroplasty induce long-term changes on the human gut microbiome contributing to fat mass regulation. Cell Metab. 2015 Aug 4;22(2):228-38. doi: I0.10I6 / j.cmet.2015.07.009

70. Aron-Wisnewsky J, Clément K, Nieuwdorp M. Fecal microbiota transplantation: A future therapeutic option for obesity/diabetes? Curr Diab Rep. 2019 Jun 27;19(8):5I. doi: | 0.1007 / s | |892-0|9-I |80-z

\section{CORRESPONDENCIA:}

Roberto E.Vigil-Guerrero

roberto.vigil.g@upch.pe

Fecha de recepción: 17-09-2021.

Fecha de aceptación: 05-I|-202I.
Contribución de los autores:Vigil-Guerrero RE: Idea del estudio, revisión de artículos, análisis e interpretación de los datos, redacción del resumen, versión final aprobada. Gonzales GF: idea del estudio, revisión crítica y versión final aprobada Gasco M: revisión de artículos, revisión crítica y versión final aprobada.

Conflicto de interés: Los autores declaran no tener cualquier relación, condición o circunstancia que pueda reducir la objetividad en la interpretación de la revisión.

Financiación: los autores han financiado íntegramente todas las etapas de la revisión. 